COMMENTARY

\title{
Spatio-temporal dynamism of hotspots enhances plant diversity
}

Dispersal and in situ speciation are seen as the main mechanisms behind the accumulation of species in an area. Biodiversity hotspots are often composed of a particular vegetation type that occupies a discrete geographical area. Recent studies focusing on these hotspots have demonstrated the contribution of ex situ speciation to their diversity. A proportion of the extant endemics that are found in hotspots originated outside the present-day boundaries of hotspots. It is possible that historically the vegetation type that the hotspot currently occupies may have previously had a larger distribution. Geologically or climatically induced repeated fragmentation followed by re-expansion of the range of this vegetation may have acted as a species pump that may in part explain the high species diversity of hotspots. This idea is similar to that of the expansion and contraction of ranges during the Pleistocene being a causal factor in generating species diversity. However, dated molecular phylogenies indicate that much of the speciation in hotspots did not occur during the Pleistocene but instead occurred during the Tertiary. Expansion and contraction of vegetation types could, however, have taken place over greater periods of time than encompassed by the Quaternary, and we highlight examples of these here and emphasize a role for vicariance in the generation of diversity.

Biodiversity hotspots are discrete areas, defined by outstanding numbers of endemic species, as well as by excessive threats. The richness of biodiversity hotspots is even more pronounced from an evolutionary perspective, as hotspots harbour more phylogenetic diversity than predicted by endemic species alone (Sechrest et al., 2002). An important question is: where and when did this phenomenal diversity originate? An understanding of the origin of species diversity in hotspots enables us to make informed choices on conservation priorities, for example maximization of species diversity or of evolutionary diversity as expressed by branch lengths of phyloge- netic trees and in particular by the ages of nodes (Sechrest et al., 2002; Forest et al., 2007).

The delineation of hotspots is not an entirely scientifically objective enterprise, as pragmatic convenience is also taken into account. Even though most hotspots host a single predominant biome type, the origin and maintenance of diversity within them have been driven by ecological and evolutionary processes that are not exclusive to them. We believe that the interpretation of diversity patterns in hotspots needs to take into account broader spatial and temporal contexts.

The issue of spatial delimitation is readily simplified in the study of island systems. Island biodiversity hotspots, such as Madagascar, are good model systems with which to address the fundamental question of when and where lineages that are endemic to them originated. Madagascan diversity is overwhelmingly assembled by lineages that dispersed into these islands during the Cenozoic (e.g. Yoder \& Nowak, 2006), and that diversified because of the exploitation of newly available ecological opportunities. A similar pattern of colonization and ensuing diversification has been found for lupins (Lupinus) in the Tropical Andes hotspot, which, given the recent elevation of their ranges, is effectively a terrestrial island archipelago (Hughes \& Eastwood, 2006). It should be noted, however, that even in the Madagascan situation vicariance, preceded by geodispersal through land bridges, has contributed slightly to the make-up of diversity.

The pattern emerging from the historical build-up of diversity in these island hotspots is consistent with dispersal as an explanatory phenomenon for large-scale biogeographical patterns. The co-development of plate tectonic theory and cladistic biogeography during the 1960s resulted in vicariance being emphasized as the common driver of congruent biogeographical patterns. However, its explanatory power has been played down with the rise of molecular dating methods (Donoghue \& Moore, 2003). It is now rarely invoked to explain continentalscale patterns in plants, and especially in angiosperms. Its role is merely demonstrated on small geographical scales, for example in the in situ diversification within hotspots. At intermediate geographical scales, such as disjunctly distributed biomes on a continent, vicariance as an operating factor has been difficult to confirm owing to the scarcity of temporally congruent divergences in independent clades (but see Crisp \& Cook, 2007). At best, it is brought up as a possible explanation for the occurrence of species-poor, narrow endemic lineages, representing once-continuous, but currently fragmented biomes.

We believe that analytical issues, notably the difficulties in delimiting areas of endemism, have contributed to the lack of recovery of vicariance events at these intermediate geographical scales. Patterns of endemism that are congruent with relationships among areas, however they are delimited, are confounded by extinction, sympatric speciation and dispersal, and are therefore less likely to be uncovered by cladistic biogeographical analyses. Another serious drawback of methods in cladistic biogeography is the failure to incorporate the dimension of time (Donoghue \& Moore, 2003). As a result, the demise of cladistic biogeography is increasingly being reported in the literature. New analytical approaches are appearing, in the context of island biogeography, that give dispersal primacy over vicariance (e.g. Sanmartín et al., 2008).

We claim, however, that for non-island biotas, where the majority of biodiversity hotspots are found, the relative contribution of vicariance processes to the regional diversity has probably been underestimated. One approach to the recovery of vicariance would be to track the congruence between distributional patterns, phylogeny and the timing of diversification on the one hand and geological processes on the other (Crisp \& Cook, 2007). A few papers using such an approach have recently appeared, demonstrating the importance of repeated vicariance for the construction of the high 
endemic diversity in hotspots, in particular in the Mediterranean Basin (Mansion et al., 2008; Yesson et al., 2009) and the two East African hotspots (Couvreur et al., 2008).

Ex situ species pumps driven by allopatric divergence have increased endemic species richness in both of these areas. For Cyclamen (Yesson et al., 2009) and Araceae (Mansion et al., 2008) in the Mediterranean, and for Annonaceae in East Africa (Couvreur et al., 2008), there is congruence between dates of divergence and geological processes. The area now recognized as the Mediterranean hotspot repeatedly joined with and separated from the current Anatolian microplate (i.e. Turkey and part of the Caucasus). The repeated connectionsdisconnections were driven by retreats and re-invasions of the Tethys Sea, setting off species pumps between the Late Eocene and the Late Miocene. These vicariance processes have been driving speciation patterns in animals in a similar way (Mico et al., 2009).

There is also evidence indicating that the East African coastal and montane hotspots were repeatedly connected to and disconnected from the Guineo-Congolian wet tropical forests. Each origin of endemic East African lineages in Annonaceae coincides with geological events that had a direct impact on climate. More precisely, they marked the start of periods of renewed aridity in East Africa, considered to have caused the breaking up of the pan-African rain forest. Thus, there is a strong congruence between the division of the African rain forest blocks and east-west divergences in Annonaceae, which therefore are in all probability identifiable as vicariance events. In more complex geodynamical settings, such as in the Mediterranean, recovering such a one-to-one relationship between vicariance events and speciation is harder. Nevertheless, the general congruence between periods of intense geodynamic activity and patterns in the timing of speciation is strong.

A static notion of hotspots, namely one of hotspots as a fixed background against which diversification patterns are studied, as opposed to one that takes into account flexible boundaries, will prevent a complete understanding of the generation of their biodiversity. Of course, past distributions of habitats can be traced only when the lineages that have been tracking these habitats have not been wiped out by extinction. The chances that vanished (corners of) hotspots of plant diversity can be traced using fossils, as in marine hotspots, are negligible. The studies discussed here show that changes in the boundaries of hotspots can be reconstructed by using clades that are good proxies for biomes, such as Annonaceae for tropical rain forests, thus showing the importance of ex situ speciation (from the perspective of the hotspot area). On intermediate geographical scales, we may see the recovery of more cases of Earth- and climate-driven processes. We anticipate the appearance of more studies showing diversification patterns that are congruent with geological mechanisms, and congruent among independent lineages, demonstrating the importance of vicariance events. These studies might go some way to challenging the common notion of biodiversity hotspots as isolated islands.

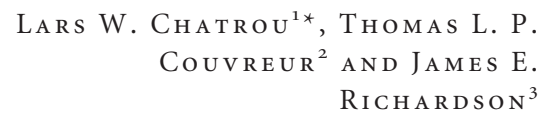

${ }^{1}$ Nationaal Herbarium Nederland, Wageningen branch, and Wageningen UR, Biosystematics Group, Generaal Foulkesweg 37, 6703 BL Wageningen, The Netherlands

${ }^{2}$ The New York Botanical Garden, 200th St. and Kazimiroff Blvd, Bronx, NY 10458-5126, USA

${ }^{3}$ Royal Botanic Garden Edinburgh, 20A Inverleith Row, Edinburgh EH3 5LR, UK E-mail: Lars.Chatrou@wur.nl

\section{REFERENCES}

Couvreur, T.L.P., Chatrou, L.W., Sosef, M.S.M. \& Richardson, J.E. (2008) Molecular phylogenetics reveal multiple Tertiary vicariance origins of the African rain forest trees. BMC Biology, 6, 54.

Crisp, M.D. \& Cook, L.G. (2007) A congruent molecular signature of vicariance across multiple plant lineages. Molecular Phylogenetics and Evolution, 43, 1106 1117.

Donoghue, M.J. \& Moore, B.R. (2003) Toward an integrative historical biogeo- graphy. Integrative and Comparative Biology, 43, 261-270.

Forest, F., Grenyer, R., Rouget, M., Davies, T.J., Cowling, R.M., Faith, D.P., Balmford, A., Manning, J.C., Procheş, Ş., van der Bank, M., Reeves, G., Hedderson, T.A.J. \& Savolainen, V. (2007) Preserving the evolutionary potential of floras in biodiversity hotspots. Nature, 445, 757 760.

Hughes, C. \& Eastwood, R. (2006) Island radiation on a continental scale: Exceptional rates of plant diversification after uplift of the Andes. Proceedings of the National Academy of Sciences USA, 103, 10334-10339.

Mansion, G., Rosenbaum, G., Schoenenberger, N., Bacchetta, G., Rosselló, J.A. \& Conti, E. (2008) Phylogenetic analysis informed by geological history supports multiple, sequential invasions of the Mediterranean basin by the angiosperm family Araceae. Systematic Biology, 57, 269-285.

Mico, E., Sanmartín, I. \& Galante, E. (2009) Mediterranean diversification of the grass-feeding Anisopliina beetles (Scarabaeidae, Rutelinae, Anomalini) as inferred by bootstrap-averaged dispersal-vicariance analysis. Journal of Biogeography, 36, 546-560.

Sanmartín, I., van der Mark, P. \& Ronquist, F. (2008) Inferring dispersal: a Bayesian approach to phylogeny-based island biogeography, with special reference to the Canary Islands. Journal of Biogeography, 35, 428-449.

Sechrest, W., Brooks, T.M., da Fonseca, G.A.B., Konstant, W.R., Mittermeier, R.A., Purvis, A., Rylands, A.B. \& Gittleman, J.L. (2002) Hotspots and the conservation of evolutionary history. Proceedings of the National Academy of Sciences USA, 99, 2067-2071.

Yesson, C., Toomey, N.H. \& Culham, A. (2009) Cyclamen: time, sea and speciation biogeography using a temporally calibrated phylogeny. Journal of Biogeography, 36, 1234-1252.

Yoder, A.D. \& Nowak, M.D. (2006) Has vicariance or dispersal been the predominant biogeographic force in Madagascar? Only time will tell. Annual Review of Ecology, Evolution, and Systematics, 37, 405-431.

Editor: José María Fernández-Palacios 